and 6 roots were affected there was impairment down the lateral side of the arm and forearm. Of the mixed cases only two (in which the deltoid was involved) showed some sensory impairment over the skin on the outer side of the arm, and the case previously mentioned in which there was impairment over the lateral side of both forearms. In the other patients no sensory impairment could be found.

\section{Special Investigations}

As many of these cases have been seen some time after the onset, detailed investigations in the acute stage have been infrequent, but when they have been possible the C.S.F., blood count, and E.S.R. have been normal, and Spillane also found this in his cases. The only exception was the finding of 10 lymphocytes per c.mm. in the C.S.F. in the case of glandular fever with radicular involvement.

Prognosis is difficult to determine in view of the varying times after the onset at which these cases have been examined. The prognosis in the serratus magnus palsies is on the whole poor: of the 21 cases in which this muscle has been involved 3 were partial from the beginning, and of these one had shown definite improvement after three months, while the other two were apparently stationary in the same period. Of the complete cases, 1 recovered completely in four months, while 5 had shown partial recovery in periods ranging from three to seven months; the remaining cases still had complete palsies when last examined at periods ranging from three to eighteen months after the onset.

Once marked atrophy has occurred in a muscle the prospect of recovery is poor; when localized atrophy is only slight, considerable and even complete recovery may occur, though the onset of recovery may be delayed for as long as six months and possibly more. In a few cases recovery has started within three to four weeks and has progressed rapidly.

\section{Pathogenesis}

There has as yet been no pathological material available from cases of this disorder; it appears, on clinical grounds, that the condition can affect single peripheral nerves, nerve roots, or combinations of these. Riddoch (1944) has suggested that the anterior horn cells may be involved in some cases, basing this view on the rapid muscular wasting which may be present and on the scattered nature of the muscular involvement which is occasionally seen. No exactly comparable condition seems to occur in the lower limbs.

The best name for the disorder is uncertain in the absence of a known aetiology or pathology. Acute brachial neuritis is already used for a different clinical syndrome, and the name applied by Spillane of "localized neuritis around the shoulder girdle" is hardly comprehensive enough. For these reasons the term "acute brachial radiculitis" has been applied to it for the time being till more is known about its aetiology.

The differential points from anterior poliomyelitis, to which it bears some clinical resemblance, have been admirably summarized by Spillane, the main ones being: the absence of signs of general disturbance in the acute stage; the normal C.S.F. when this has been examined early in the illness; the sensory impairment in a number of the cases; and the occurrence of these cases in the absence of typical cases of poliomyelitis in the same district at the same time. To these may be added the fact that it may occur at the age of 50a most unusual happening with poliomyelitis.

The aetiology of the condition is still speculative, and the somewhat mixed group of apparently precipitating causes has not helped on this point. It has been suggested that a virus infection is the cause: the main point against this is the absence of fever or other signs of general infection and the normal C.S.F. findings. In most diseases due to neurotropic viruses-even in zoster, in which the main incidence of the infection is on the posterior root ganglia-an increase of cells is found in the C.S.F.

There is one condition which has a very close clinical resemblance to it, and that is serum neuritis (Kennedy, 1929 ; Young, 1932). This may occur after the injection of any form of serum, usually about the seventh to ninth day; there are frequently the usual symptoms of serum sickness, followed within a day or two by severe pain in the region of the shoulder and upper arm; muscle weakness shows within the next few days, and when this happens the severe pain passes off. The paralysis which may be accompanied by sensory changes is most often in the distribution of the fifth and sixth roots or the circumflex nerve, but at times paralysis of the serratus magnus may arise. The exact cause of these cases is uncertain, but it is thought that oedema of the affected nerves occurs as a reaction to the foreign protein in the serum. I think that the possibility of some, as yet uncertain, aetiological relationship between serum neuritis and acute brachial radiculitis must be considered before assuming that a meurotropic virus is the causative agent.

With the lack of knowledge of the aetiology, treatment can be only palliative-analgesics for the pain in the acute stage, support for paralysed muscles to prevent stretching, and active and passive exercises and electrical stimulation of the affected muscles when the painful stage has passed.

\section{Summary}

The syndrome of pain around the shoulder, followed in the course of a few days by muscular weakness and wasting and at times sensory changes, is described.

The anatomical distribution of the weakness can be a single peripheral nerve, two or more spinal roots, or combinations of these.

A number of apparent precipitating factors are mentioned, the most outstanding being operation for hernia in 8 of the 36 cases.

The possible aetiology is discussed and an analogy drawn between this condition of acute brachial radiculitis and serum neuritis.

My thanks are due to Major-Gen. Mitchiner, C.B.E., for permission to publish this paper; to Brig. Riddoch for help in its production; and to Major P. S. Buckley, R.A.M.C., for the use of notes of cases under his care.

\section{REFERENCES}

Kennedy, F. (1929). Amer. J. med. Sci., 177, 555 Richardson, J. S. (1942). Lancet, 1, 618 .

Riddoch, G. G. (1944). Personal communication. Spillane, J. D. (1943). Lancet, 2, 532.

Young, F. (1932). J. Amer. med. Ass., 98, 1139

\section{THE TRANSNASAL INTRAGASTRIC RYLE'S TUBE IN TROPICAL PRACTICE}

\section{A LIFE-SAVING MEASURE}

$\begin{array}{ll} & \text { BY } \\ \text { G. A. RANSOME } & \text { L. M. GUPTA } \\ \text { Lieut.-Col., } \text { I.A.M.C. } & \text { Capt., I.A.M.C. }\end{array}$

\section{J. C. S. PATERSON}

$$
\text { Capt., R.A.M.C. }
$$

Ryle's tubes have been used by us in a series of 355 acute medical cases during the past year. So far as can be ascertained, this represents the first extensive trial, in tropical practice, in which the method has been applied to the problems of hydration and alimentation presented by such acute cases. Its use was introduced, in the first place, to meet the special problem of the dehydrated patient who would not or could not drink that amount of fluid considered necessary for him. Dehydration, among coolies especially, was seen on a scale rarely encountered in civil practice, and in many cases was so gross as to resemble that of cholera. Marriott's (1943) teaching of the fatuity of what he calls "the homoeopathic dosage of a pint or two " is wasted unless we realize that in gross dehydration the fluid loss is to be measured in gallons. Furthermore, some of these patients refused to drink, and spat out the water or saline offered to them. In a series of 30 of our. cases this symptom was found to be associated with a low serum chloride figure, and possibly represents a vicarious form of water intoxication. In the more robust patients this can be corrected by the intravenous injection of one or more pints of hypertonic $(2 \mathrm{~N})$ saline solution, after which the previously non-drinking dehydrated patient will drink avidly. Other patients, however, are often so enfeebled by disease on admission to hospital that for them to drink is too much effort. It is in such cases that the tube becomes a life-saving instrument, since by its use the body fluid may be restored in a few hours. 
About five pints of normal saline solution can be given to these patients by slow intravenous drip in a 24 -hour period, but this represents only a fraction of the fluid loss. To give more by the intravenous route, which has been tried, exposes the patient to the very grave risk of pulmonary oedema, particularly if there is any central circulatory weakness and if the serum proteins are low. On the other hand, a large quantity of fluid can safely be absorbed from the alimentary canal when administered through the Ryle's tube.

Appreciation of the advantage of this method after a short trial led to the extension of its use to include practically every type of case whose state of hydration was in any. respect inadequate or in which fluids required to be forced. Such were the following cases: malaria and blackwater fever, cerebral malaria (over 100 cases), cerebrospinal meningitis (65 cases), enteric fever and typhus, pneumonia, psychoses, and coma due to other causes--e.g., head injuries.

\section{Advantages of the Nasal Route}

1. When in situ Ryle's intragastric tube is comfortableindeed, not felt.

2. Food, fluids, and drugs may be given as necessary, independently of the patient's desires. Unpleasant-tasting drugs such as quinine and paraldehyde may be given without distress, and are retained.

3. In lung cases the respiration is not embarrassed.

4. Adequate fluid intake can be ensured, and its quantity accurately measured and recorded. The quantity given may be large and yet without the risk of producing pulmonary oedema. In gross dehydration the fluid required may be as much as 10 to $\cdot 15$ pints. In relation to this it is worthy of note that, although water absorbed from the alimentary canal is followed by a speedy diuresis, physiological saline does not have this effect. Further, saline absorbed from the alimentary canal is better retained and produces a less immediate diuresis than does saline given direct into the blood stream (Wright, 1940).

\section{Technique of Passing the Tube}

Cases may be divided into three groups: (1) those with a swallowing reflex even in the presence of coma; (2) those without a swallowing reflex-i.e., cases in deep coma ; (3) refractory patients-e.g., maniacal and cerebral irritation cases. The first essential is to test the tube for patency and leaks by syringing a little water through it. Lubrication of the tube is all-important, and the best means is with liquid paraffin, although vaseline or olive oil may be used. With apprehensive or non-cooperative patients analgesia of the nose and soft palate may be induced by $5 \%$ percaine nasal spray, but this has not been necessary in military practice. It is desirable to sit up the patient and then proceed as follows:

Group 1.-Pass the tube into the nostril, choosing the one which is the more patent, and gently push it backwards along the floor of the nasopharynx. The passage past the turbinates is assisted by tilting up the tip of the nose with the finger. Now pass 6 to 8 in. of the tube, when the patient will cough; wait until the inevitable retching occurs, followed by a swallow, which will draw the tube into the oesophagus; after this it may be rapidly pushed down to the third mark on the tube. Swallowing may be aided by instructions to the patient, by water to drink, or by placing something palatable on the tongue. There is no danger of getting the tube into the larynx if the act of swallowing is awaited before passing it onward.

Group 2.-Prop up the patient and lean him forward. Put two fingers into the mouth and the posterior pharynx, using a mouth-gag if necessary. Pass the tube through the nostril and into the pharynx as before, and use the fingers to guide it behind the epiglottis into the oesophagus. It is then pushed onward as before. A tube passed into the trachea speaks for itself. Prove that the tube is in situ by aspirating a little of the gastric contents.

Group 3.- Refractory patients may be dealt with by either of two methods. One is to anaesthetize with an intravenous barbiturate (evipan sodium) just short of abolishing the swallowing reflex. If this is abolished, wait until it returns and then proceed as in Group 1. When the tube is in the stomach put in a dose of potassium bromide gr. 40 , chloral hydrate gr. 20 , with or without tinct. opii mm. 20 , or paraldehyde drs. 2. The altermative method is to produce twilight sleep with intramuscular sodium luminal or subcutaneous scopolamine-morphine before attempting to pass the tube. By employing these methods with judgment unseemly struggles are avoided.
With a little practice it is very easy to become really proficient at passing an intragastric Ryle's tube. Three or four successes make anyone confident. It is important to train as many as possible to do this. Our nursing officers and trained nursing sepoys soon do it with ease.

\section{Fixation of the Tube}

Bring the free end alongside the nose and up to the forehead between the eyes, and fix it with strapping. When not in use the free end should be tied or be closed with a clip. Some patients, especially psychotics, tend to pull out the tube at first. This may be overcome by splinting the arms to prevent flexion of the elbow and tying to the bed if necessary, and/or giving sedatives through the tube. Patients, however, soon become accustomed to it, and are scarcely aware of its presence. Recently, a Chinese patient with a delightfully indolent attitude towards life became extremely annoyed when his tube was removed and he once more had to rouse himself for food and drink.

The period during which the tube is in situ is governed by the condition and progress of the patient. It varies between 12 hours and three or four days. One of our patients, a psychotic, had a tube in place for three weeks without ill effect or fouling of the tube. Fluids and feeds may be given either as a continuous drip, using a graduated flask and Murphy drip apparatus, or by means of a funnel without drip connexion. The former method is preferable in cases of coma, since the rate of absorption proceeds pari passu with the rate of drip, and regurgitation is thus avoided.

\section{Possible Drawbacks}

In discussing this method with Brig. H. L. Marriott, Consulting Physician, India Command, and Col. G. F. Taylor, Consulting Physician, Eastern Army, two possible objections to its use were raised-namely, the possibility of ulceration of the laryngopharynx, and the danger of bronchopneumonia arising from the aspiration of gastric contents regurgitated alongside the tube. Consequently, in 19 cases treated by this method which ultimately came to necropsy these ill effects were sought, but were never found. In coma the method of drippingin fluid minimizes the risk of regurgitation.

Serum proteins have been estimated in some 300 cases of varying type coming under our care, and in an alarming proportion of these low albumin figures have been observed, especially among coolie personnel. Care must be taken not to force the fluid to excess in these, as in a few instances a generalized anasarca has resulted. Constant reference to a "fluid intake-output chart," which we always keep in our wards, forms a valuable guide to the fluid balance.

\section{Discussion}

Although this method has been employed previously in surgical practice for continuous gastric drainage and in head injuries (McAlpine, 1944), and in medical practice for the continuous duodenal drip feeding in peptic ulceration and recently during the Bengal famine (Indian Research Fund Association, 1944), its value is by no means so well known as it deserves to be. Recent graduates from the medical schools of Bombay, Madras, Calcutta, Patna, and Lahore are unfamiliar with its application to acute medicine, and as these have been interns, we can only assume that either the method is not used in the teaching institutions of India or its use has been sporadic.

One of us, with five years' postgraduate experience of acute medicine in teaching hospitals in London and four in Singapore, regards the full application of this method, forced on him by the exigencies of war, as a considerable advance in treatment, and, had he only been earlier aware of its full possibilities, believes that certain patients who died in the Medical College Hospital in Singapore would have been saved by its bold use. Particularly has it solved the problem of hydration in malarial coma, in which, owing to the reduction of serum albumin brought about by the disease itself, and frequently a preexisting condition as well, the risk of pulmonary and secondary cerebral oedema following intravenous saline infusion is very real, as has been widely appreciated in the past (Thomas and Sydenstricker, 1940). 
A most important lesson we have learnt is that no patient is too ill to have a tube passed. Even seemingly moribund dehydrated cases of pneumonia and meningococcal meningitis have been saved by this method. These patients were so ill that had one not had previous experience of the intragastric drip one would not have dared to pass a tube. Brinton (1941) draws attention to severe dehydration developing during cerebrospinal fever, and mentions the method of giving fluid by means of a nasal catheter, but does not convey to the reader that an intragastric tube is used, and states that an adult should take 2 to 3 pints of fluid every 24 hours. This demonstrates the difference in tropical practice, in which, in the same disease, a fluid intake limited to 3 pints a day would lead to disaster not only from dehydration but commonly in the form of sulphapyridine haematuria. We have in these cases used Ryle's tube with such complete success for the administration of large quantities of fluid, and also sulphapyridine, that to pass a tube in cerebrospinal fever is now our routine procedure.

It was with some trepidation that this method was first employed in acute pulmonary disease, but the need for fluid was so great that it was attempted, the intravenous route being out of the question and the rectal " not satisfyingly certain" (Bailey, 1939). It was not found appreciably to embarrass respiration-rather less so than do many forms of oxygen apparatus in common use. An especial advantage is that hydration proceeds during sleep.

The attitude of our nursing staff, drawn from all parts of the British Commonwealth, has been one of unqualified approval, since it saves much of their time spent in repeatedly requesting the patient to drink. The tube achieves what may only otherwise be cone by special day and night sisters under nursing-home conditions.

It is realized that this practice is not as familiar as it should be, and we are certain that an extension of its use and emphasis on the quantity of fluid needed will be found to save the lives of many people whom one would ordinarily expect to die in a few hours.

Our thanks are due to Col. F. R. Cawthorn, I.A.M.C., for the facilities given to us in developing this technique and for permission to publish this article. We also wish to acknowledge the valuable and willing co-operation of many nursing and medical officers.

\section{REFERENCES}

Bailey, H. (1939). Pye's Surgical Handicraft, 11th ed., Wright and Sons, Bristol Brinton, D. (1941). Cerebrospinal Fever, Livingstone and Co., Edinburgh.

Indian Research Fund Association (1944). Report of Inquiry into Effects of Starvation, Indian med. Gaz, 79, 74

McAlpine, D. (1944). Indian Physician, 3, 61.

Marriott, H. L. (1943). Proceedings of Conference of Medical Specialists of Eastern Army.

Thomas and Sydenstricker (1940). Quoted in Manson's Tropical Diseases, 11th ed., Cassell and Co., London.

Wright. Samson (1940). Applied Physiolog.1, 7th ed., London.

\section{SUPRARENAL HAEMORRHAGE REPORT OF A CASE}

BY

\section{J. Williams, M.B., Ch.B.} AND

RONALD ELLIS, M.D., M.R.C.P.

Hon. Asst. Physician, Royal Liverpool United Hospital

Below we report a case of suprarenal haemorrhage diagnosed during life. The clinical data are best presented as day-to-day details.

\section{Case History}

The patient was an unmarried woman, aged 23 , and before the onset of symptoms was apparently in excellent health. The illness began on a Saturday, during the height of the influenza epidemic last winter, but the patient did not send for her doctor until the following Monday.

Saturday.-Onset of malaise, slight sore throat, short dry cough, and one attack of vomiting.

Sunday.-Main complaint now was of "feeling cold." The throat was still sore, but there was in addition a complaint of pain in the lumbar region. This pain was never severe, and was not referred to the region of the loins. The symptom she stressed especially was the persistent feeling of cold.
Monday.- She called in one of us, and was seen for the first time at 3 p.m. The story of the preceding days was elicited, and in addition she complained of frontal headache, and was found to be menstruating. The menstrual period was to time, but the loss was excessive. The symptoms of the previous day were still present, but were no worse. On examination temperature $100^{\circ}$; pulse 84 , with fair volume, regular and apparently normal tension; fauces and pharynx injected, but no gross clinical tonsillitis and no exudate. There was no cervical adenopathy or coryza. The heart, lungs, and abdomen presented no abnormality. There were no signs of meningeal irritation, and no change in the optic fundi or drumheads. The skin did not show any rash and there was no sweating.

Tuesday.-During the early hours of the morning the patient complained of intense cold and a feeling of profound fatigue. She was seen by C. J. W. again at 6 p.m. The whole picture was now in marked contrast with that of the previous evening. The woman was restless and had an anxious look. She was intensely cyanosed, especially in the face and lips, hands and forearms, feet, and legs up to the knees. The temperature was $96^{\circ}$ and there was a profuse cold sweat. The pulse rate was 120 , regular, but of poor volume; respirations 30, with frequent sighings. Examination of lungs, heart, and abdomen revealed nothing further. Central nervous system: Mentally clear and co-operative; cranial nerves normal, optic fundi normal, pupils dilated but reacting normally; knee- and ankle-jerks absent; arm-jerks present but weak; plantar response flexor. The arms and legs could be moved only with difficulty. Light touch, pin-prick, heat, and cold were blunted over the extremities, but deep sensation was normal.

At 8 p.m. the same day the woman's condition was obviously worse. The cyanosis was deeper, and there was no pulse at the wrist; she was mentally apathetic but rational, and was still sweating profusely.

At 11 p.m. she was seen in consultation. The main findings were as above : the heart rate was now about 160 or over, and the clinical state was obviously deteriorating rapidly. At this stage a tentative diagnosis of suprarenal haemorrhage was made, and adrenaline, pituitrin, strophanthin, etc., were administered. No cortical extract was immediately available. The patient died three hours later, at 2 a.m. on the Wednesday morning, after an illness lasting about four days.

Post-mortem Examination.-The right adrenal was completely disrupted by haemorrhage, which had burst through the capsule of the gland. The left adrenal showed discrete haemorrhagic areas throughout its substance. The other organs were macroscopically normal. There was no haemorrhage into serous membranes or any splenic enlargement. The necropsy was performed under difficulties, but was reasonably complete.

This case presented great diagnostic difficulties. As mentioned previously, it occurred at the height of an influenza epidemic, with fresh cases of influenza being seen hourly, and not unnaturally the diagnosis when first seen was influenzaa diagnosis which may have been correct. But the striking change for the worse which occurred between the first and second visits of C. J. W. obviously called for a further diagnosis. The clinical picture was one of profound circulatory collapse, the cause of which an examination failed to reveal.

The unusually heavy menstrual period suggested the possibility of an abortion, and the question of poisoning by some drug taken to procure abortion was discussed. Hospital investigation was out of the question; the patient lived in a lonely farmhouse, and the weather was so foggy that she could be visited only with the greatest difficulty. At the midnight consultation it seemed that the only diagnosis which would account for the profound asthenia was acute suprarenal failure: This very tentative diagnosis was confirmed by the necropsy.

The literature of acute suprarenal haemorrhage has become quite extensive, though the number of recorded cases, so far as we have been able to discover, does not exceed about 60 : owing to wartime difficulties our search of the literature has not been as complete as we would wish, and we are open to correction on this point. By far the greater number of recorded cases and discussions concern the now wellrecognized Waterhouse-Friderichsen syndrome, due in the majority of cases to meningococcal septicaemia, the symptomatology being characterized by sudden onset, malaise, restlessness, often vomiting and diarrhoea, followed by cardiovascular collapse with intense cyanosis, often a striking purpuric eruption, and death within 24 hours. But deviations from the syndrome have been recorded by many writers, and 